\title{
Smart high-performance materials for the multi-hazard protection of civil infrastructure
}

\author{
T. B. Messervey ${ }^{1}$, D. Zangani ${ }^{1} \&$ S. Casciati $^{2}$ \\ ${ }^{1}$ D'Appolonia S.p.A., Genova, Italy \\ ${ }^{2}$ ASTRA Department, University of Catania, Italy
}

\begin{abstract}
High-performance materials can be utilized to provide infrastructure protection against multiple threats and to simultaneously satisfy the needs of fields that are typically considered independently. For example, the reinforcement of an existing or newly built masonry structure with sensor-embedded textiles and nanoparticle-based mortars provides increased strength and ductility against earthquake, improved fragmentation/ballistic properties against blast, and inservice data to conduct structural health monitoring, life-cycle management performance prediction, and incident emergency assessment for first responders. Viewed independently, any such benefit/action may not be considered cost effective. However, viewed in a multi-hazard perspective, such actions are potentially synergistic and cost saving. This paper describes ongoing industrial research at D'Appolonia toward the realization of smart high-performance materials with embedded informatics capabilities. Key technological barriers to be solved are rugged sensors and sensing systems that can survive extreme conditions as well as the probabilistic methods necessary to characterize the utility of such products in monetary terms. Progress in these activities through ongoing research projects is detailed.
\end{abstract}

Keywords: multifunctional materials, multi-hazard protection, risk.

\section{Introduction}

The safe and reliable performance of civil infrastructures such as buildings, bridges, dams, and transportation networks are of critical importance as it directly impacts the sustainable economic growth, productivity, and social well being of a modern society [1]. To this end, comparisons across countries in 
varying stages of development can be used to show that Gross Domestic Product (GDP), life expectancy, and infrastructure development are highly correlated. As a result, society relies on its engineers and government to design, maintain, and regulate structures that are safe and perform as intended over their service lives. Furthermore, it should be emphasized that it is also an area with very little margin for error. Several notable infrastructure failures which include the failure of the New Orleans levee system during Hurricane Katrina (2005), the Minneapolis I35W bridge collapse (2007), the Laval highway overpass collapse in Canada (2006), and the Northeast powergrid blackout in the United States due to a falling tree branch (2003) have shown that the public quickly loses confidence in its engineers, infrastructure managers, and government when infrastructures fail. Although such reactions may overlook the complexity, difficulty, and magnitude of the problem at hand, the bottom line is that structures should not fail and result in the loss of life, especially if the technology and ability to detect and address structural deficiencies exists. In terms of magnitude, new civil engineering construction is the largest industry in the world representing approximately $10 \%$ of annual GDP. Of this $10 \%$ of GDP spending, an estimated $5-10 \%$ is the result of the failure (not necessarily collapse) of existing structures [2]. For most countries, existing structures and civil infrastructure are their most valuable assets and their upkeep represents one of their most significant investments.

Civil infrastructure is vulnerable to damage from several sources which include design error, construction error, overloading, misuse, natural hazards, accident, deliberate attack, and most commonly, deterioration with aging. Over the past several decades, design codes, inspection programs, and management programs have evolved as the body of knowledge has advanced with respect to the treatment of uncertainty and technological advancements have enhanced computational methods [3]. In addition and in response to these challenges, several fields of study and professional societies have formed which include the structural health monitoring (SHM) community, the life-cycle management community (LCM), researchers concerned with risk-based decision making and risk-based approaches, and the field of security. Although research is beginning with respect to the integration of the SHM and LCM [4], work in each of these respective areas is typically conducted independently.

An interesting and appropriate area of research is the consideration and quantification of the utility of new technologies and or processes in a multihazard perspective. For example, the hardening or retrofit of critical structural elements can decrease a structure's vulnerability to natural hazards, increase its level of security, and lower its life-cycle maintenance costs. In addition and in particular, SHM offers a powerful mechanism to reduce uncertainty across these domains. This paper introduces and examines a novel multifunctional material being developed in the EU research project POLYTECT (Polyfunctional Technical Textiles Against Natural Hazards) in a multi-hazard perspective. Computational methods appropriate for the characterization of the utility of such materials in a multi-hazard perspective are identified. Lastly, upcoming field tests to implement and evaluate these materials and approaches are detailed. 


\section{Multifunctional materials in construction and retrofit}

Materials with embedded sensing capabilities offer a new and interesting mechanism to collect structural data. The advantage of such materials is that they can be manufactured off-site, quality control can be conducted, and upon installation they become integrated with the structure. These advantages correlate to less on-site labour, sensors that are better protected, and a higher probability that the sensors will perform as intended over their service life [5]. The European research project POLYTECT (Polyfunctional Technical Textiles Against Natural Hazards) is one such effort to develop multifunctional materials through the development of fibre optic sensor-embedded textiles.

Fibre optic sensors are well suited for integration in textile manufacturing processes and they have many advantages compared to electrical and other sensing methods. They can operate in strong electromagnetic fields and in harsh environments such as high temperature, explosive and chemical environments as well as nuclear radiation [8]. Because the optical fibres are small, they can be installed and can measure in places inaccessible for other sensors. In addition, these small fibres can be bundled to record different measurement types within the same cable bundle (e.g. temperature, moisture, and strain). Lastly, optical fibres can be installed continuously over distances of many kilometres allowing for distributed sensing. In such cases, the fibre optic sensor provides measurements at any position along the fibre for the value of interest (e.g. temperature, strain, pressure or nuclear radiation) and as such only one fibre optic sensor can substitute for thousands of local electrical, electronic or other point-wise measuring devices and costs can be reduced.

The primary advantage of these textiles is that they are multifunctional, they provide both reinforcement and monitoring functions targeted for masonry and geotechnical applications. For masonry structures, increases in strength of up to $200 \%$ and increases in ductility of up to $250 \%$ are being recorded in the laboratory testing of medium-scale walls. To date, over 21 sensor-embedded textile patterns have been manufactured and tested by industrial partners. This has involved the design of textile patterns, the adaptation of industrial warpknitting machines to embed fibre optic sensors into the textile, laboratory testing of the manufactured products, numerical modelling of product use, and preliminary field tests [6]. Several project highlights are shown in Fig. 1 which includes two representative sensor-embedded textile products (a geotextile filter mat and a typical reinforcing grid) and the testing of a large-scale wall reinforced with sensor-embedded textiles at the University of Karlsruhe, Germany.

Applications for such textiles are numerous and diverse across the fields of security, seismic engineering, SHM, and LCM. With respect to the monitoring function, the collected information can be utilized to ensure safe construction conditions, to assess in-service performance, to schedule inspection, maintenance, and repair activities, to conduct performance-based design, and eventually to provide quantitative information that enables the improvement of existing codes and guidelines. With respect to the reinforcing function, the application of the textiles reduces structural vulnerability to all types of hazards 
through increases in strength and ductility. Fig. 2 shows several applications for POLYTECT being demonstrated through field tests within the project. They include the monitoring and reinforcement of an active fault on a roadway embankment at a coal mining site, the monitoring of construction activities and the in-service performance of a railway embankment, and the reinforcement and monitoring of a river embankment project along the Danube River in Romania. Not pictured but planned are the monitoring and reinforcement of areas prone to slope stability failures in mountainous zones and the chemical and performance monitoring of a landfill to optimize its performance as a bio-reactor and to guard against chemical leakages. In particular, the reinforcement and monitoring of the roadway embankment fault was notably successful. In this case it was determined that the fault was growing at approximately $2 \mathrm{~mm}$ per day. This enabled a slope stability analysis and the mine owner repaired the road prior to any loss of mine operating hours or accident due to a road failure [7].

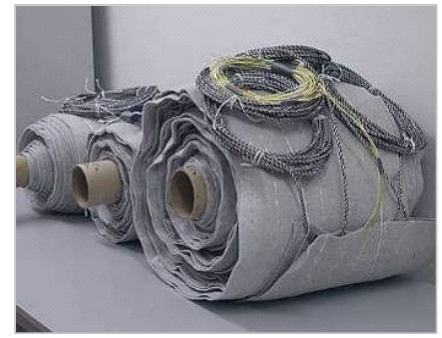

(a)

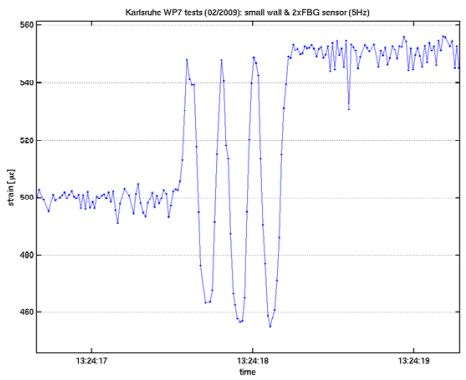

(c)

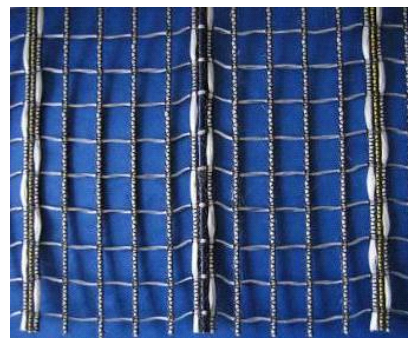

(b)

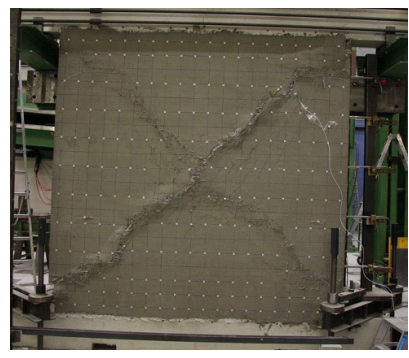

(d)

Figure 1: (a) Sensor-embedded geotextiles, (b) a sensor-embedded textile grid pattern, (c-d) testing on a large-scale masonry wall at the University of Karlsruhe, Germany.

For each application, selecting the appropriate fibre optic sensor type and interrogation technique requires the consideration of measurement range, spatial resolution, strain accuracy, measurement acquisition time, and cost. Fibre Bragg Grating (FBG) optical fibres offer the possibility for high frequency measurements but are limited point or semi-distributed measurements (using multiple FBGs) that record the average displacement along the gage length. 
Instead, Optical Time Domain Reflectometry (OTDR) or Brillioun scattering signal processing techniques provide fully distributed sensing but with higher system costs and slower data acquisition times. With respect to the fibre types, glass optical fibres (GOF) and plastic optical fibres (POF) are available. GOF have the advantage of being more developed and readily available, but may be fragile with respect to the construction environment and service range of civil infrastructures. POF have the distinct advantage that they can undergo up to $40 \%$ strain, but acquisition techniques are less developed and measurement ranges are still relatively short (e.g. hundreds of meters for POF vs. kilometers for GOF).
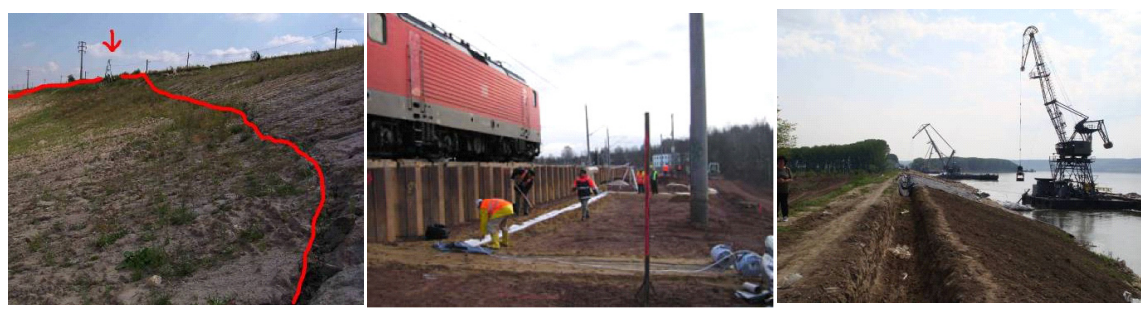

Figure 2: From left to right, the monitoring of a fault of concern at a roadway embankment, the monitoring of a railhead embankment, and the monitoring of a river embankment project (courtesy of Gloetzl Corporation, Germany, STFI, Germany, and Iridex Construction, Romania).

\section{Assessing the utility of multifunctional materials in a life-cycle context}

Developing and leveraging the use of monitoring technologies for civil applications requires insight, planning, and continued research. The rapid pace of advances in monitoring technologies provides a sharp contrast when compared to the time required to affect changes in civil engineering, a field governed by laws, codes, time-tested experience, and where projects themselves may span decades. Although SHM offers great potential, it should be anticipated that such technologies will not be adopted unless they are proven cost-effective due to competing resource demands from the backlog of required maintenance and rehabilitation activities. As such, metrics and methods that calculate and communicate the costs and benefits associated with monitoring must be identified and employed so that alternatives may be adequately compared.

Because monitoring data is inherently probabilistic, probabilistic methods are required to incorporate this information into engineering analyses. Furthermore, in order to adequately quantify the utility (e.g. the cost benefit in monetary terms) of increased information, reduced uncertainty, and the corresponding increased level of safety provided by real-time in-service data, the consideration of risk and reliability methods is required. If the process is considered over time, a life-cycle cost analysis is necessary. For an existing structure, this implies 
reliability-based life-cycle management approaches with the inclusion of risk. For a new structure, this implies performance-based and durability-based design.

The minimum expected life-cycle cost with respect to lifetime performance is the most widely used criterion for design optimization of a new structural system. The general form of the expected life-cycle cost can be calculated as [9]

$$
C_{E T}=C_{T}+C_{P M}+C_{I N S}+C_{R E P}+C_{F}
$$

where $C_{E T}=$ expected total cost, $C_{T}=$ initial design/construction cost, $C_{P M}=$ expected cost of routine maintenance, $C_{I N S}=$ expect cost of performing inspections, $C_{R E P}=$ expected cost of repairs and $C_{F}=$ expected cost of failure. A comparison of eqn (1) calculated with and without the application of multifunctional materials (and to include their life-cycle costs) can be utilized to assess the utility of these materials over the structure's lifetime. The most difficult term to calculate in such an analysis is $C_{F}$ (or the risk cost) which in its most simple form is

$$
\text { Risk }=R=C_{F}=p_{f} C
$$

where risk is calculated as the product of the likelihood of an event, $p_{f}$, and the associated consequences in monetary terms, $C$, given the event occurs. Structures are inherently subjected to different risks or hazards. These include, amongst others, the ones related to the probability of failure due to seismic events $p_{f S}$, extreme loads $p_{f L}$, incident $p_{f I}$, and deliberate attack $p_{f A}$. Under the simplified assumption that these events can only occur independently from each other, the total risk to be considered in comparing alternatives with and without multifunctional materials can be expressed as

$$
\text { Total Risk }=\left(p_{f S}+p_{f L}+p_{f I}+p_{f A}\right) C
$$

where the probabilities of failure account for the seismic, loading, incident, and attack probabilities of failure respectively. The concept is to characterize the risks relevant to the problem being analyzed.

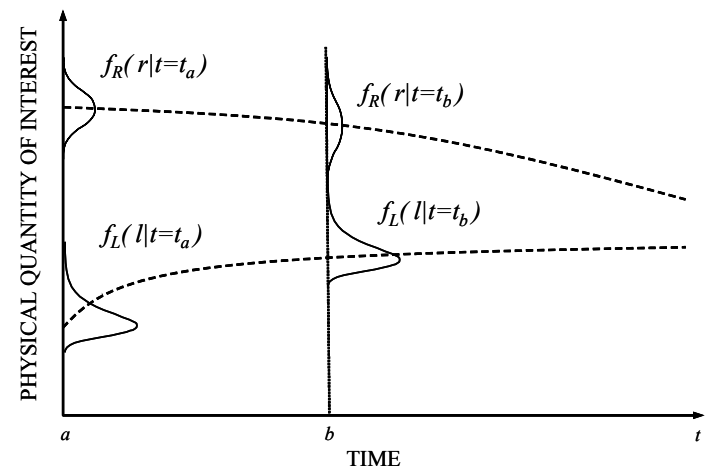

Figure 3: $\quad$ Trace of the structural resistance $R$ and load effect $L$ in a time dependent reliability analysis.

Methods to calculate these probabilities of failure are available in each of the associated fields but are rarely considered together. In some cases, tables and checklists are utilized to simplify the problem (common for incident risk) and in 
other cases detailed structural analyses are carried out. It is also important to properly account for time effects. Over time, a structure's ability to safely carry loads (e.g. their resistance $R$ ) deteriorates and the likelihood of encountering a load $L$ of greater magnitude increases as shown in Fig. 3 . The result is that a time dependent analysis must be carefully structured to calculate the cumulative probability of failure for the time period of interest using the correct load and resistance terms which can also include conditioning on past safe performance and use of a hazard function [10].

\section{Assessing seismic retrofit using the PEER approach}

One probabilistic formulation of estimating the utility of retrofit actions of particular interest to an upcoming experiment within the POLYTECT project is the PEER (Pacific Earthquake Engineering Research Center) approach. Focused primarily on seismic evaluations and originally proposed by Cornell and Krawinkler [11], this approach disaggregates the problem into four components or models which are hazard, structural response, structural vulnerability, and loss. In this framework, the theorem of total probability can be applied three times to conditional probabilities relating the four model components to find the complementary CDF of the decision variable $D V$ as [11]

$$
\begin{aligned}
\lambda(D V)= & \iiint G(D V \mid D M) \cdot|d G(D M \mid E D P)| \\
& \times|d G(E D P \mid I M)| \cdot|d \lambda(I M)|
\end{aligned}
$$

where $D M$ is the damage measure(s), $E D P$ is the structural response(s) termed the engineering demand parameter, $I M$ is an intensity measure(s) of the seismic hazard, and $\lambda$ is an occurrence rate typically taken as the mean annual occurrence rate using a Poisson process for the intensity measure. The decision variable $D V$ translates the damage into values useful to the decision maker which can be economic losses, out-of-service time, casualties, or can be identified with a limit state of the structure [12].

Fig. 4 shows the upcoming experiment of interest for this approach which will be conducted at Institute of Mechanics of Materials and Geostructures (IMMG) SA near Athens, Greece. The structure will be constructed, tested, and destroyed four times to evaluate different reinforcing strategies with the sensorembedded textiles. Historical earthquake time histories will provide realistic load demands. Aside from evaluating POLYTECT materials, the goal of the experiment is to find ways to cost effectively reduce the vulnerability of typical masonry structures located in the Mediterranean Basin.

With respect to this experiment, the PEER approach is selected for its treatment of uncertainty, its segregation of the problem into its parts, and for the ability to communicate with other researchers using a standardized approach. For the tests at IMMG, it will be possible to correlate the structural response (EDP), damage level (DM), and intensity measures IM) as the structure is progressively brought to failure for both the reinforced and unreinforced cases. 

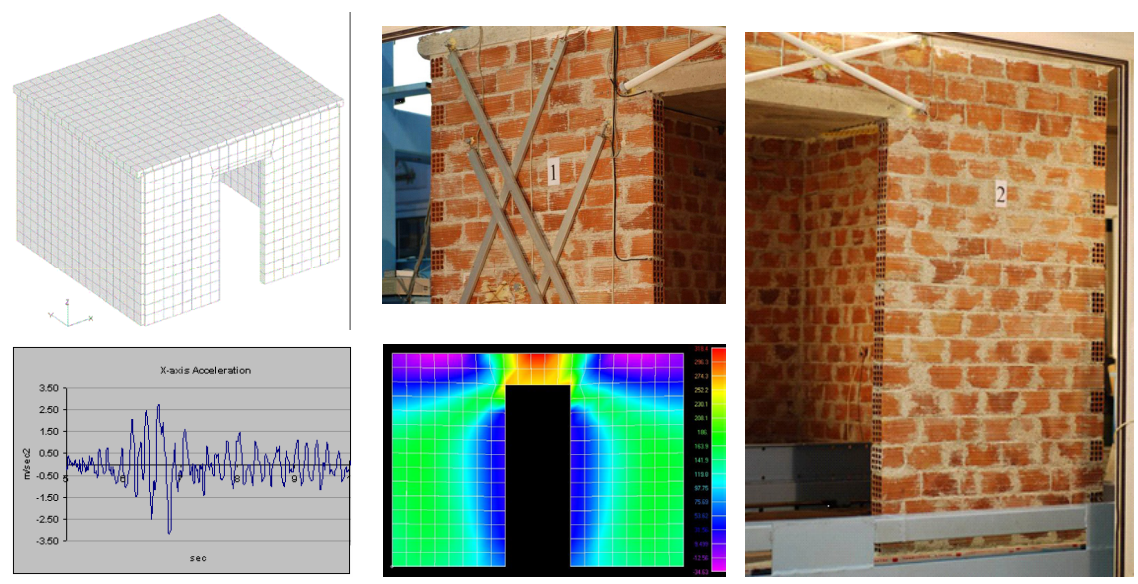

Figure 4: Model, earthquake excitation, and structure for reinforcement and seismic testing at IMMG near Athens, Greece.

Several simplifications of the general (or full) PEER approach as shown in eqn (4) are available [13] and will be investigated for use. With this background, the following initial steps are planned to use the masonry shaking table field tests as an opportunity to begin forming Polytect specific tools that incorporate risk and reliability concepts using the PEER approach:

- Select an appropriate engineering demand parameter EDP that can be obtained via fibre optic sensors placed on the outer walls.

- Select an appropriate earthquake intensity measure IM. Vary this measure during testing.

- Correlate damage $D M$ to the $E D P$ and $I M$ for both the reinforced and unreinforced structures.

- Select cost as the decision variable $D V$.

- Obtain $I M$ for representative zones beginning with the Mediterranean basin.

- Apply the PEER approach calculations to determine the difference in the decision variable between the unreinforced and reinforced structures.

\section{Conclusions}

Sensor-embedded multifunctional materials offer a novel and important product for the construction sector. Such materials can be utilized to increase infrastructure protection against multiple threats and to simultaneously satisfy the needs of fields that are typically considered independently. Several important benefits include the opportunity to conduct performance-based design, 
the ability to assess structures using site-specific data, and the ability to manage structures using less uncertain information. Despite these benefits, it is unlikely that such products will be adopted unless they are shown to be cost effective. As such, methods and metrics appropriate for probabilistic analysis, the inclusion of risk, and reliability approaches must be considered within a life-cycle context. For the engineer conducting such analyses, a multi-hazard perspective should be conducted to best quantify the risk reduction associated with increased information, reduced uncertainty, and an increased level of performance provided by real-time in-service data across multiple fields of interest.

\section{Acknowledgements}

The partial funding support of European Grant NMP2-CT-2006-026789 in support of the Polytect project is gratefully acknowledged. In addition, the contribution of the companies and research institutions performing this work is acknowledged and especially those directly in support of the work mentioned in this paper which include Glötzl, Iridex, Selcom, BAM, STFI, CETMA, IMMG, Safibra, the University of Karlsruhe, Germany, and the University of Kassel, Germany.

\section{References}

[1] Frangopol, D. M., Liu, M. Life-cycle cost and performance of civil structures. McGraw-Hill 2006 Yearbook of Science and Technology, McGraw-Hill, New York, pp. 183-185, 2006.

[2] Bijen, J. Durability of Engineering Structures: Design, Repair and Maintenance. Woodhead Publishing Limited, Abington Hall, Cambridge, England, 2003.

[3] Frangopol, D.M. \& Messervey, T.B. Maintenance principles for civil structures. Chapter 89 in Encyclopedia of Structural Health Monitoring. Ed. C. Boller, F.K. Chang, and Y. Fujino, Vol 4. John Wiley \& Sons, Ltd., UK, pp. 1533-1562, 2009.

[4] Messervey, T.B. \& Frangopol D.M. Integration of health monitoring in asset management in a life-cycle perspective. Proceedings of the Fourth International Conference on Bridge Maintenance and Safety, Seoul, Korea, July 13-17, 2008, IABMAS'08.

[5] Messervey T.B. \& Zangani D. Monitoring Technologies: Progressing from theory to application. Proceedings of the 4th International Conference on Smart Structures and Materials, 13-15 July, Porto, Portugal, 2009.

[6] Messervey T.B. \& Zangani D. Industrial Smart Material Applications: The Polytect Project and its Expansion into the IMS program. Proceedings of the $4^{\text {th }}$ International Conference on SHM of Intelligent Infrastructures (ISHMII09), 22-24 July, Zurich, Switzerland, 2009.

[7] Liehr, S., Lenke, P., Wendt, M., Krebber, K., Glotzl, R., Schneider-Glotzl, J., Gabino, L., \& Krywult, L. Distributed polymer optical fiber sensors in geotextiles for monitoring of earthwork structures. Proceedings of the $4^{\text {th }}$ 
30 Safety and Security Engineering III

International Conference on SHM of Intelligent Infrastructures (ISHMII09), 22-24 July 2009, Zurich, Switzerland, 2009.

[8] Krebber, K. Protection of Critical Infrastructure Using Fibre Optic Sensors. International Newsletter on Micro-Nano Integration (MST-News). No. 4/07, pp. 12-14, 2007.

[9] Frangopol, D.M. Application of life-cycle reliability-based criteria to bridge assessment and design. P.C. Das (ed.), Safety of Bridges. Thomas Telford, London, pp 151-157, 1997.

[10] Frangopol, D.M. \& Messervey, T. Risk assessment for bridge decision making. Proceedings of the Fourth Civil Engineering Conference in the Asian Region, CECAR 4, Taipei, Taiwan, June 25-28, 2007 (invited paper); in ASCE Tutorial \& Workshop on Quantitative Risk Assessment, Taipei, Taiwan, June 25-28, 2007, 37-42, 2007.

[11] Cornell, A. \& Krawinkler, H. Progress and challenges in seismic performance assessment. PEER Center News 3(2): 1-3, 2000.

[12] Augusti, G. \& Ciampoli, M. Performance-Based Design in risk assessment. Probabilistic Engineering Mechanics, 23(4) pp. 496-508, 2008.

[13] Haukaas, S. Unified reliability and design optimization for earthquake engineering. Probabilistic Engineering Mechanics, Ed. M. Shinozuka \& P.D. Spanos, 23(4) pp. 471-581, 2008. 